\title{
Tailored terahertz pulses from a laser-modulated electron beam
}

\author{
Michael C. Martin, John Byrd, Zhao Hao, David Robin, Fernando Sannibale, Robert W. Schoenlein, \\ Alexander Zholents, Max Zolotorev
}

Advanced Light Source, Lawrence Berkeley National Laboratory, Berkeley, CA, USA
e-mail: MCMartin@lbl.gov

Interaction of an electron beam with a femtosecond laser pulse co-propagating through a wiggler modulates the electron energies within a short slice of the electron bunch comparable with the duration of the laser pulse (Figure 1). Propagating around an electron storage ring, this bunch develops a longitudinal density perturbation due to the dispersion of electron trajectories. Figure 1 shows how this creates femtosecond electron bunch 'wings' which are used for femtosecond x-ray light. In addition, this density perturbation emits temporally and spatially coherent tera-hertz pulses which are inherently synchronized to the modulating laser. This gives us a new way to study coherent synchrotron radaition, and creates an opportunity for tuning the $\mathrm{THz}$ emmission specifically for the needs of a given experiment.


Figure 1. Schematic of the 'laser slicing' setup at the ALS.

We have measured the intensity and spectra of these pulses for the first time at the Advanced Light Source. We present and analyze spectral measurements as a function of beam parameters (current and momentum compac-tion), and from


Figure 2. Slicing density distributions for nominal (red) and high-alpha lattices (blue), at two beam ports. beam ports close to and far from the slicing wiggler. These measure-ments are compared to the predicted emission for each beam parameter and port location (Figures 2 and 3). We then describe a technique which allows the terahertz pulse to be tailored by appropriate modulation of the laser pulse. This type of technology will enable coherent control experiments using this novel coherent $\mathrm{THz}$ source.



Figure 3. Time domain signal of the far-IR bolometer detector output showing the intense emissions every 1 ms, coincident with the slicing laser.

We show that the growth of a microbunching instability which creates high-power $\mathrm{THz}$ emissions can be triggered by the laser slicing and our initial understanding of this intriguing phenomenon.
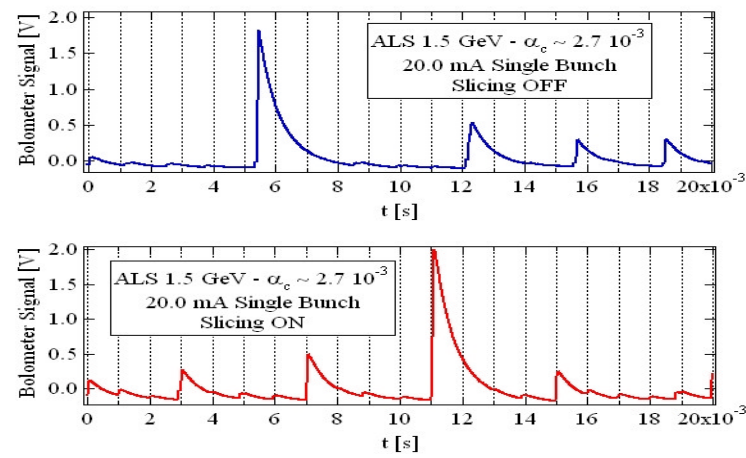

Figure 4. "Bursting" emissions (upper panel) become synchronous to the laser slicing (lower panel). 


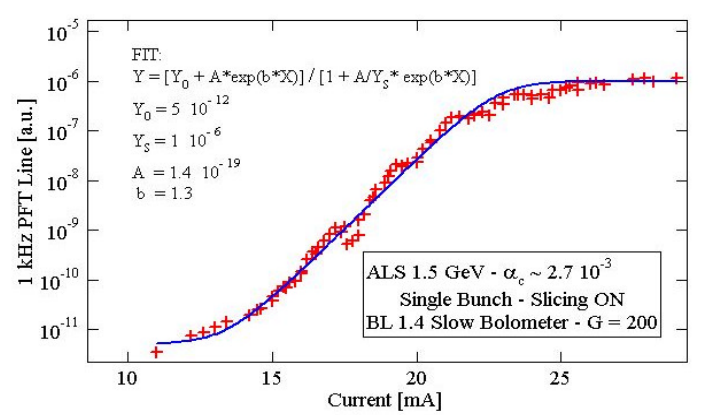

Figure 5. Stimulated bursting emission as a function of beam current grows exponentially followed by a saturation.

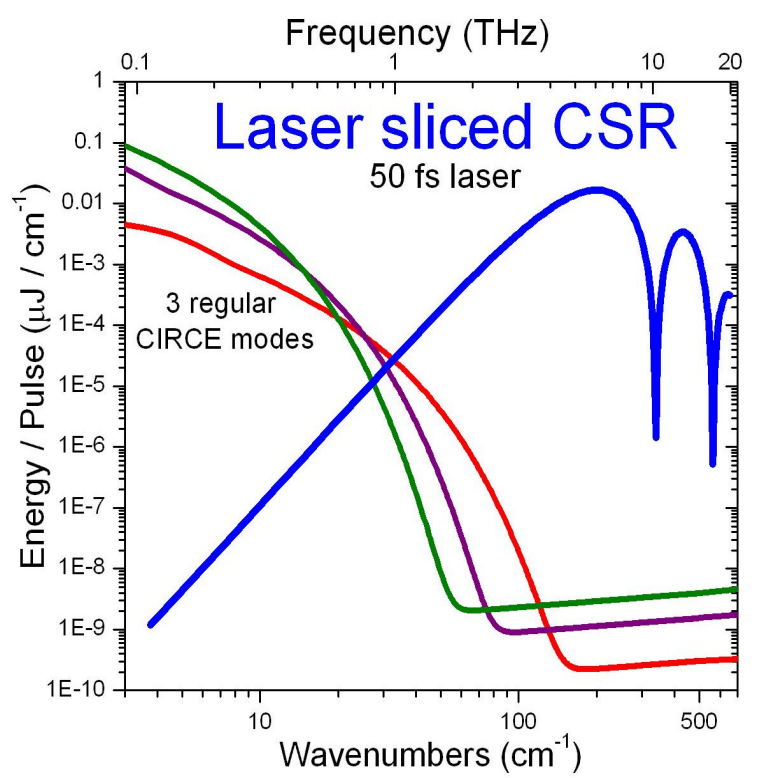

Figure 6. Laser slicing $\mathrm{THz}$ output from an optimized CSR source such as the proposed CIRCE project in Berkeley.

Finally, we show the predicted output from laser slicing on an optimized CSR source such as the proposed CIRCE source at Berkeley. http://CIRCE.lbl.gov/ Such a source would produce high intensity single cycle pulses up to $20 \mathrm{THz}$ with efield strengths of $1 \mathrm{MV} / \mathrm{cm}$ on a sample which opens up the possibility for many non-linear scientific applications. 